\title{
Standardizing Outcome Reporting in Bariatric/Metabolic Surgery
}

\author{
Mikael Victorzon ${ }^{1}$
}

Published online: 18 August 2015

(C) Springer Science+Business Media New York 2015

There is a variety in how outcomes following bariatric and metabolic surgery are reported, especially concerning postoperative morbidity. The Dindo-Clavien (D-C) classification [1] of postoperative morbidity has gained wide acceptance in all fields of surgery and is used also by some authors in their outcome reports of bariatric/metabolic surgery, but not by all. Most studies report only less than 30 days morbidity leaving more long-term morbidity unreported. The lack of agreement on the definition of a surgical complication and what constitutes an actual surgical complication add to the confusion. This makes comparison of results difficult. Recently, the D-C classification was criticized by the clinical issue committee of the American Association for Metabolic and Bariatric Surgery (ASMBS) for not taking the time frame into account [2]. This committee recommended the use of a simplified

Mikael Victorzon

mikael.victorzon@vshp.fi

1 Department of Surgery, University of Turku, Vaasa Central Hospital, Hietalahdenkatu 2-4, 65130 Vaasa, Finland classification based on dividing morbidity in minor and major, divided further by the time frame in early and late morbidity with cutoff of 30 days. In my opinion, the classification of postoperative morbidity following bariatric surgery should not differ from the classification widely used following any other kind of surgery as doing so would only add to the confusion. However, I agree that the time frame is important and strongly encourages everyone to take time into account but to otherwise use the D-C classification.

Conflict of Interest The author declares that he has no competing interests.

Ethical Approval This article does not contain any studies with human participants or animals performed by any of the authors.

\section{References}

1. Dindo D, Demartines N, Clavien PA. Classification of surgical complications: a new proposal with evaluation in a cohort of 6336 patients and results of a survey. Ann Surg. 2004;240:205-13.

2. Brethauer SA, Kim J, El Chaar M, et al. Standardized outcomes reporting in metabolic and bariatric surgery. Obes Surg. 2015;25: $587-606$. 